\title{
Ceratectomia fotorrefrativa com mitomicina-C personalizada pela frente de onda corneana para 0 tratamento de hipermetropia após ceratotomia radial
}

\author{
Corneal wavefront-guided photorefractive keratectomy with mitomycin-C for \\ consecutive hyperopia after radial keratotomy
}

\author{
Ramon Coral Ghanem ${ }^{1}$ \\ Emir Amin Ghanem² \\ Newton Kara-José ${ }^{3}$
}

Estudo realizado no Hospital de Olhos Sadalla Amin Ghanem - Joinville - Santa Catarina (SC) - Brasil.

${ }^{1}$ Oftalmologista do Hospital de Olhos Sadalla Amin Ghanem - Joinville (SC) - Brasil. Pós-graduando do Departamento de Oftalmologia da Faculdade de Medicina da Universidade de São Paulo - USP - São Paulo (SP) - Brasil.

${ }^{2}$ Oftalmologista do Hospital de Olhos Sadalla Amin Ghanem - Joinville (SC) - Brasil.

${ }^{3}$ Livre Docente, Professor Titular do Departamento de Oftalmologia da Universidade de São Paulo - USP São Paulo (SP) - Brasil.

Endereço para correspondência: Ramon Coral Ghanem. Rua Fernando de Noronha, 225 - Apto. 901 - Joinville (SC) CEP 89203-072

E-mail: ramonghanem@gmail.com

Recebido para publicação em 11.06.2009

Aprovação em 17.11.2009

Os autores declaram não possuir interesse financeiro nos produtos referidos no estudo. Não houve auxílio financeiro para realização deste estudo.

Nota Editorial: Depois de concluída a análise do artigo sob sigilo editorial e com a anuência do Dr. Renato Ambrósio Jr. sobre a divulgação de seu nome como revisor, agradecemos sua participação neste processo.

\begin{tabular}{|c|}
\hline RESUMO \\
\hline 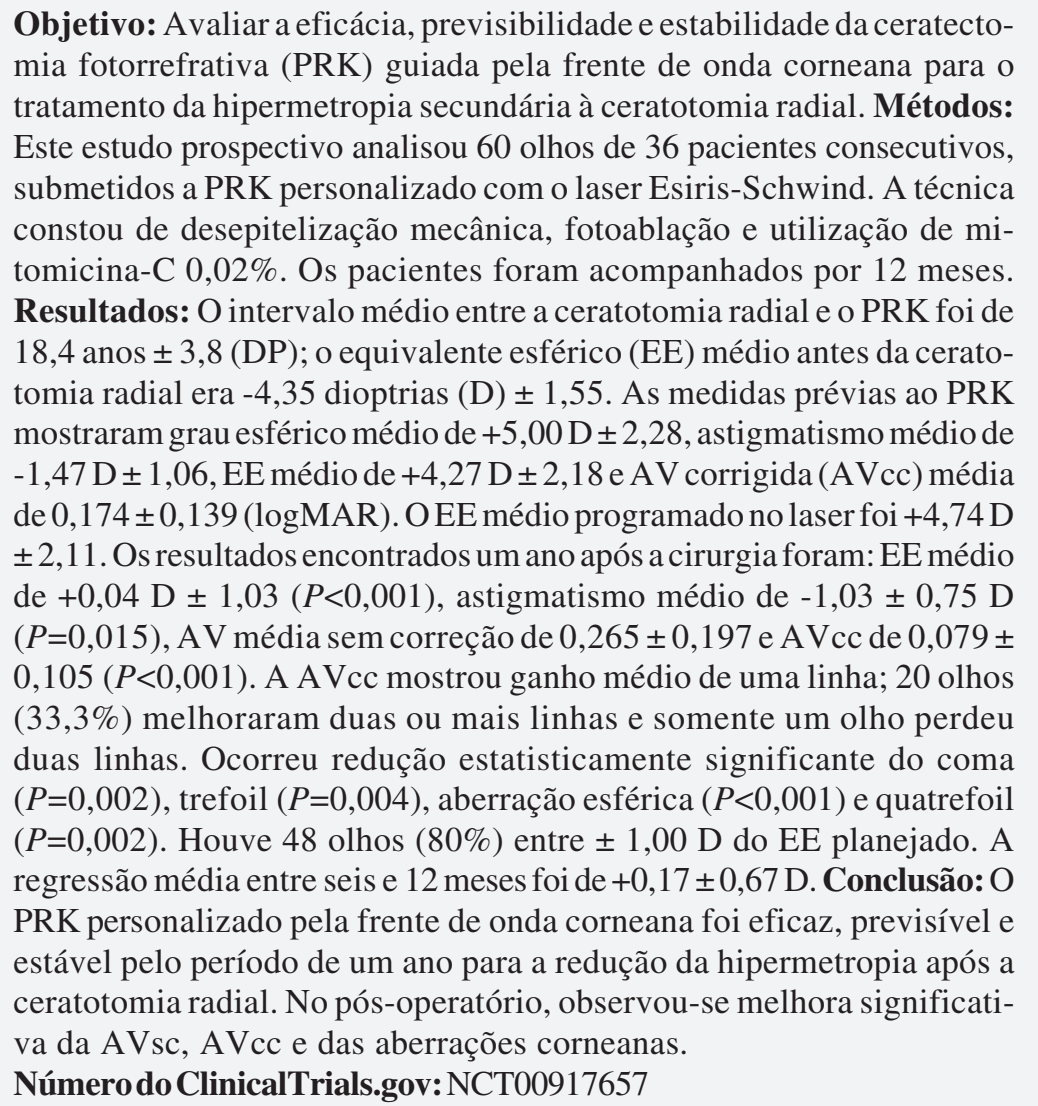 \\
\hline
\end{tabular}

Descritores: Ceratectomia fotorrefrativa; Mitomicina; Ceratotomia radial; Hipermetropia; Astigmatismo

\section{INTRODUÇÃO}

A ceratotomia radial (CR) foi uma técnica para a correção de miopia utilizada na década de 80 e no início da década de 90. Com bisturi de diamante, eram realizadas incisões radiais de aproximadamente $95 \%$ da espessura corneana, nas regiões periférica e mediano-periférica. As incisões causavam enfraquecimento dessas regiões, que, principalmente sob a ação da pressão intraocular, evoluíam com encurvamento periférico e consequente aplanamento central, corrigindo a miopia. 
Em 1994, foram publicados os resultados do estudo clínico prospectivo multicêntrico PERK (Prospective Evaluation of Radial Keratotomy Study), realizado para avaliar a CR. Foi demonstrado que, após dez anos da cirurgia, 53\% dos olhos alcançaram acuidade visual sem correção (AVsc) de 20/20 e $85 \%$, de $20 / 40$ ou melhor ${ }^{(1)}$. Apesar da boa eficácia do procedimento, entre seis meses e dez anos após a cirurgia, $43 \%$ dos olhos haviam sofrido desvio hipermetrópico maior ou igual a 1 dioptria (D), com incremento anual de $5 \%$, sem tendência à estabilização ${ }^{(1)}$. Estes resultados demonstraram que os pacientes tratados com CR naquele período continuaram a sofrer as consequências do procedimento sem nunca alcançar estabilidade, com sintomas que incluem, além da hipermetropia progressiva, dificuldade de visão noturna por halos e ofuscamento, perda da sensibilidade de contraste, flutuação diária da visão e diminuição da resistência corneana a traumas ${ }^{(1-2)}$. Além disso, essa população hoje, em quase sua totalidade, apresenta presbiopia, o que limita a acomodação mesmo em pequenos graus de hipermetropia ${ }^{(3-4)}$.

As opções de correção para os casos de hipermetropia secundária à CR são muito restritas, visto que o uso de óculos geralmente não fornece AV satisfatória, pois não corrige o astigmatismo irregular e as aberrações de alta ordem, principalmente a aberração esférica ${ }^{(2-4)}$. As lentes de contato rígidas representam a melhor opção para corrigir esses erros refrativos, estabilizar a visão e reduzir os sintomas relacionados com as aberrações, entretanto sua adaptação pode ser bem mais difícil porque, além dos problemas técnicos causados pela limitação de desenhos para córneas obladas, muitos pacientes optaram pela cirurgia devido à intolerância a seu uso ${ }^{(5)}$.

Várias técnicas cirúrgicas já foram utilizadas para as hipercorreções após CR, dentre elas as mais importantes são a sutura em bolsa de Grene ${ }^{(6)}$, o "laser in situ keratomileusis" (LASIK) e a ceratectomia fotorrefrativa (PRK).

Resultados visuais favoráveis foram inicialmente descritos com o LASIK. Para tratamento de hipermetropia, a eficácia (AVsc de 20/40 ou melhor) variou entre 65\% e 90\% ${ }^{(7-13)}$. Ocorreram várias complicações: abertura das incisões radiais durante o levantamento do retalho, crescimento epitelial na interface, ceratite lamelar difusa e ectasia corneana iatrogênica ${ }^{(7-15)}$.

Técnicas de ablação de superfície, como o PRK, já foram utilizadas para tratar hipo e hipercorreções após $\mathrm{CR}^{(3-4,16-19)}$. Estudos prévios realizados no Brasil demonstraram bons resultados com a ablação guiada pela topografia para hipercorreções após $\mathrm{CR}^{(3-4)}$. Sabe-se, entretanto, que o risco de "haze" após PRK é maior em olhos com cirurgias prévias, como CR, LASIK e transplante de córnea, indicando-se, assim, o uso profilático de mitomicina-C (MMC) no intraoperatório ${ }^{(3-4,20-22)}$.

Este estudo tem por objetivo avaliar os resultados do PRK com MMC personalizado pela frente de onda corneana derivada da topografia (Corneal Wavefront, CORWAVE ${ }^{\circledR}$ ) para o tratamento de hipermetropia e astigmatismo após CR.

\section{MÉTODOS}

\section{Desenho do estudo e pacientes}

Foi realizado estudo clínico prospectivo, incluindo 60 olhos de 36 pacientes operados consecutivamente, submetidos a CORWAVE-PRK para tratamento de hipermetropia após CR, no Hospital de Olhos Sadalla Amin Ghanem, entre outubro de 2005 e fevereiro de 2008.

Todas as cirurgias foram realizadas por três cirurgiões (EAG, VCG, RCG), utilizando a mesma técnica, em protocolo padronizado. Os pacientes assinaram um termo de consentimento que explicava o objetivo do estudo proposto, suas alternativas e possíveis complicações. Este estudo foi aprovado pela Comissão de Ética para Análise de Projetos de Pesquisa da Faculdade de Medicina da Universidade de São Paulo-SP.

\section{Critérios de inclusão e exclusão}

Foram incluídos neste estudo pacientes com:

- Hipermetropia ou astigmatismo hipermetrópico com equivalente esférico até $+9,25 \mathrm{D}$ e astigmatismo até $-4,5 \mathrm{D}$;

- Idade maior do que 30 anos;

- AVsc de 20/40 ou pior;

- AV com correção (AVcc) de 20/60 ou melhor.

Foram excluídos os pacientes que apresentavam doenças oculares ou sistêmicas que pudessem comprometer o resultado cirúrgico ou afetar a $\mathrm{AV}$ pós-operatória e aqueles dos quais não foi possível obter topografia reprodutível. Também foram excluídos pacientes com cirurgias fotoablativas corneanas prévias.

\section{Avaliação pré-operatória}

Foi realizado exame ocular completo. A análise topográfica e de frentes de onda corneanas (Corneal Wavefront) foi realizada com o aparelho Keratron Scout. Realizou-se também tomografia corneana com Orbscan IIz e paquimetria ultrassônica corneana.

\section{Técnica cirúrgica}

O procedimento seguiu a seguinte ordem: anestesia tópica e antissepsia da região periocular; marcações no limbo a 0 e 180 graus na lâmpada de fenda com caneta de violeta de genciana; remoção do epitélio corneano em sala cirúrgica, sob campos estéreis, feita com espátula romba (mecânica), nos $10 \mathrm{~mm}$ centrais. Iniciou-se pela periferia corneana a $1 \mathrm{~mm}$ do limbo, de maneira centrípeta para evitar a abertura das incisões radiais. Em seguida, realizou-se o controle manual da ciclotorção alinhando-se as marcações previamente realizadas no limbo com as da lente ocular do laser e a fotoablação personalizada.

Em todos os casos foi realizada a ablação guiada pela frente de onda corneana, tratando-se a hipermetropia total (sob cicloplegia), o astigmatismo e também as aberrações de alta ordem corneanas. Na maioria dos casos realizou-se hipercorreção da hipermetropia, tanto para induzir miopia em pacientes présbitas, levando a monovisão, como para compensar a regressão inicial do tratamento. 
Utilizou-se o laser "flying-spot" Esiris Schwind de $200 \mathrm{~Hz}$, com disparos de $0,8 \mathrm{~mm}$ de diâmetro e controle dos movimentos oculares (eye-tracking) de $330 \mathrm{~Hz}$. Com instrumentalização do software ORK-CAM (Custom Ablation Manager; versão 2.2.3.650), a fotoablação foi analisada e planejada.

Após a fotoablação, aplicou-se MMC $0,2 \mathrm{mg} / \mathrm{mL}(0,02 \%)$ com uma esponja $\left(\right.$ Merocel $\left.^{\circledR}\right)$ por 20 a 40 segundos em toda área tratada. A MMC foi aplicada por 40 segundos quando o risco de "haze" era maior, ou seja, nas ablações mais profundas do que 100 micra e em córneas submetidas a suturas prévias. A superfície foi, então, lavada com $20 \mathrm{ml}$ de solução salina balanceada e colocou-se uma lente de contato terapêutica (LCT) até a completa epitelização.

\section{Pós-operatório}

No pós-operatório aplicaram-se colírios de gatifloxacino $0,3 \%$, de cetorolaco de trometamina $0,4 \%$ e de ciprofloxacina $0,3 \%$, associada à dexametasona $0,1 \%$. Após a retirada das LCT, foram utilizados colírios lubrificante e de etabonato de loteprednol $0,5 \%$ por um mês.

As avaliações pós-operatórias incluíram análise da incidência de "haze" central ( $3 \mathrm{~mm}$ ) - e periférico, utilizando-se a escala de intensidade de 0 a $4^{(23)}$.

\section{Análise estatística}

As medidas de AV foram convertidas para $\log \mathrm{MAR}^{(24)}$. Foi aplicado o teste de Kolmogorov-Smirnov com fator de correção de Lilliefors para avaliar a normalidade das variáveis. Foram usados os testes $t$ de Student pareado para as variáveis com distribuição normal e o teste de Wilcoxon para as variáveis sem distribuição normal, entre elas as aberrações corneanas e medidas de $\mathrm{AV}$ sc e $\mathrm{AV}$ cc. Os valores $P<0,05$ foram considerados estatisticamente significantes.

\section{RESULTADOS}

\section{Pacientes e acompanhamento}

Foram estudados 60 olhos de 36 pacientes, 11 homens e 25 mulheres. A média de idade foi de $50 \pm 6,8$ anos (de 34 a 65 anos) (Tabela 1). Todos os olhos haviam sofrido CR entre $11 \mathrm{e}$ 26 anos antes de se submeter ao PRK (Tabela 2). Todos os pacientes foram avaliados após decurso de seis e de 12 meses do PRK. A espessura corneana média, medida com o paquímetro ultrassônico, foi de $560 \pm 33 \mu \mathrm{m}$ e com o Orbscan $\mathrm{IIz}^{\circledast}$, de $545 \pm 62 \mu \mathrm{m}$. Nenhum olho foi submetido a nova fotoablação (retratamento).

\section{Dados do intraoperatório}

O epitélio corneano foi completamente removido nos $10 \mathrm{~mm}$ centrais de todos os olhos, sem a abertura das incisões radiais. A profundidade média de fotoablação foi de $78 \pm 28 \mu \mathrm{m}$ (de 33 a 148) e a zona óptica variou de 6 a $6,5 \mathrm{~mm}$, com média de $6,4 \pm 0,2$. A zona total de ablação variou de 8 a $10 \mathrm{~mm}$, com média de 9,1 $\pm 0,6$. MMC foi utilizada por 20 segundos em 44 olhos $(73,3 \%)$ e por 40 segundos em 16 (26,7\%). Não foram observadas complicações intraoperatórias.

\section{Acuidade visual}

No pré-operatório, a AVsc média em logMAR foi de $0,846 \pm$ 0,428 (variando de 20/40 a conta dedos a $66 \mathrm{~cm}$ ). Seis meses após o PRK, a AVsc média foi de 0,300 $\pm 0,202$ (variando de 20/20 a 20/150) e após um ano, de 0,265 $\pm 0,197$ (variando de 20/20 a 20/150). Depois de um ano, dentre os 17 olhos $(28,3 \%)$ com AVsc pior do que 20/40, 9 (53\%) apresentavam EE miópico intencional $\geq 0,75 \mathrm{D}$ (monovisão). Observou-se melhora estatisticamente significante da AVsc no pós-operatório (Tabela 3). A figura $1 \mathrm{~A}$ demonstra a eficácia do procedimento.

\section{Resultados refrativos}

No pré-operatório, o EE médio foi de $+4,27 \pm 2,18 \mathrm{D}$ (de $+0,25$ a $+9,25$ D). Após seis meses, o EE médio foi de $-0,13 \pm$ $1,00 \mathrm{D}(\mathrm{de}-2,25 \mathrm{a}+2,25)$ e depois de um ano, $+0,04 \pm 1,03 \mathrm{D}$ (de $-1,88 \mathrm{a}+3,00$ ) (Figura 1B). A figura 2A demonstra a verificação da previsibilidade do tratamento após um ano, onde 29 olhos (48\%) estavam entre $\pm 0,5 \mathrm{D}$ do EE planejado, 48 (80\%) entre \pm 1 D e $56(93 \%)$ entre \pm 2 D. A regressão refracional média entre

\begin{tabular}{|lc|}
\hline \multicolumn{2}{|c|}{ Tabela 1. Dados demográficos $(\mathbf{n = 6 0 )}$} \\
\hline Características & Resultados \\
№ olhos / $\mathrm{n}^{\circ}$ pacientes & $60 / 36$ \\
Sexo, $\mathrm{n}(\%)$ & \\
Masculino / feminino & $11(31 \%) / 25(69 \%)$ \\
Idade (anos) & $50,0 \pm 6,8$ \\
Média \pm DP & 34 a 65 \\
(mín - máx) & $24(67 \%)$ \\
Pacientes com cirurgia bilateral, $n(\%)$ & \\
DP= desvio padrão & \\
\hline
\end{tabular}

\begin{tabular}{|c|c|}
\hline Características & Resultados \\
\hline \multicolumn{2}{|c|}{ Miopia pré-CR* (dioptrias) } \\
\hline Média \pm DP & $-4,35 \pm 1,55$ \\
\hline (mín - máx) & $-8,00$ a $-1,75$ \\
\hline \multicolumn{2}{|c|}{ Intervalo entre a CR e o PRK $^{*}$ (anos) } \\
\hline Média \pm DP & $18,4 \pm 3,8$ \\
\hline (mín - máx) & 11 a 26 \\
\hline \multicolumn{2}{|c|}{ Incisões de CR, n (\%) } \\
\hline 4 & $4(7 \%)$ \\
\hline 8 & $55(92 \%)$ \\
\hline 14 & $1(1,7 \%)$ \\
\hline \multicolumn{2}{|c|}{ Incisões transversais (astigmáticas), n (\%) } \\
\hline 0 & $37(62 \%)$ \\
\hline 1 & $7(11,7 \%)$ \\
\hline 2 & $11(18,3 \%)$ \\
\hline 4 & $5(8,3 \%)$ \\
\hline
\end{tabular}




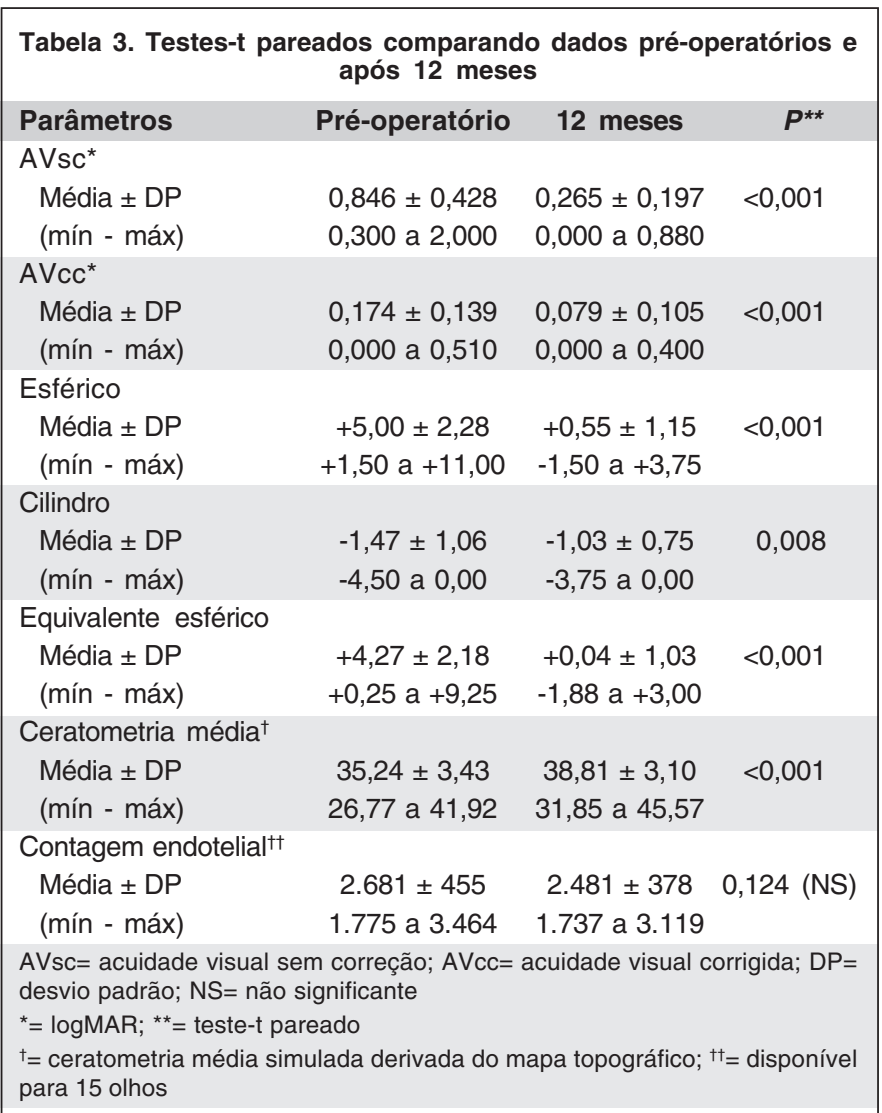

seis e 12 meses foi de $+0,17 \pm 0,66 \mathrm{D}$. Houve uma redução estatisticamente significativa do EE, grau esférico e cilíndrico no pós-operatório (Tabela 3). A figura 2B ilustra, em gráfico de dispersão, a correção planejada vs. correção alcançada.

\section{Topografia corneana}

No pós-operatório final observou-se curvatura corneana média de 38,8 \pm 3,1 D (variação 31,9 a 45,6 D), um aumento notável em relação ao pré-operatório, quando a curvatura média era de 35,2 $\pm 3,4 \mathrm{D}$ (variação 26,7 a 41,9 D) $(P<0,001$; teste-t pareado).

\section{Análise de frentes de onda corneana}

A evolução do RMS (root-mean-squared), valor P-V (peak-to-valey) e das aberrações corneanas de baixa ordem é demonstrada na figura $3 \mathrm{~A}$. Observou-se redução estatisticamente significativa do defocus ( $P=0,047$; teste de Wilcoxon). As aberrações de alta ordem corneanas podem ser observadas na figura 3B. Dentre elas houve redução estatisticamente significativa do coma $(P=0,002)$, trefoil $(P=0,004)$, aberração esférica $(P<0,001)$ e quatrefoil $(P=0,002)$.

\section{Segurança}

Após 12 meses da cirurgia, observou-se "haze" periférico grau 2 ou 3 em cinco olhos e "haze" central discreto em um olho.
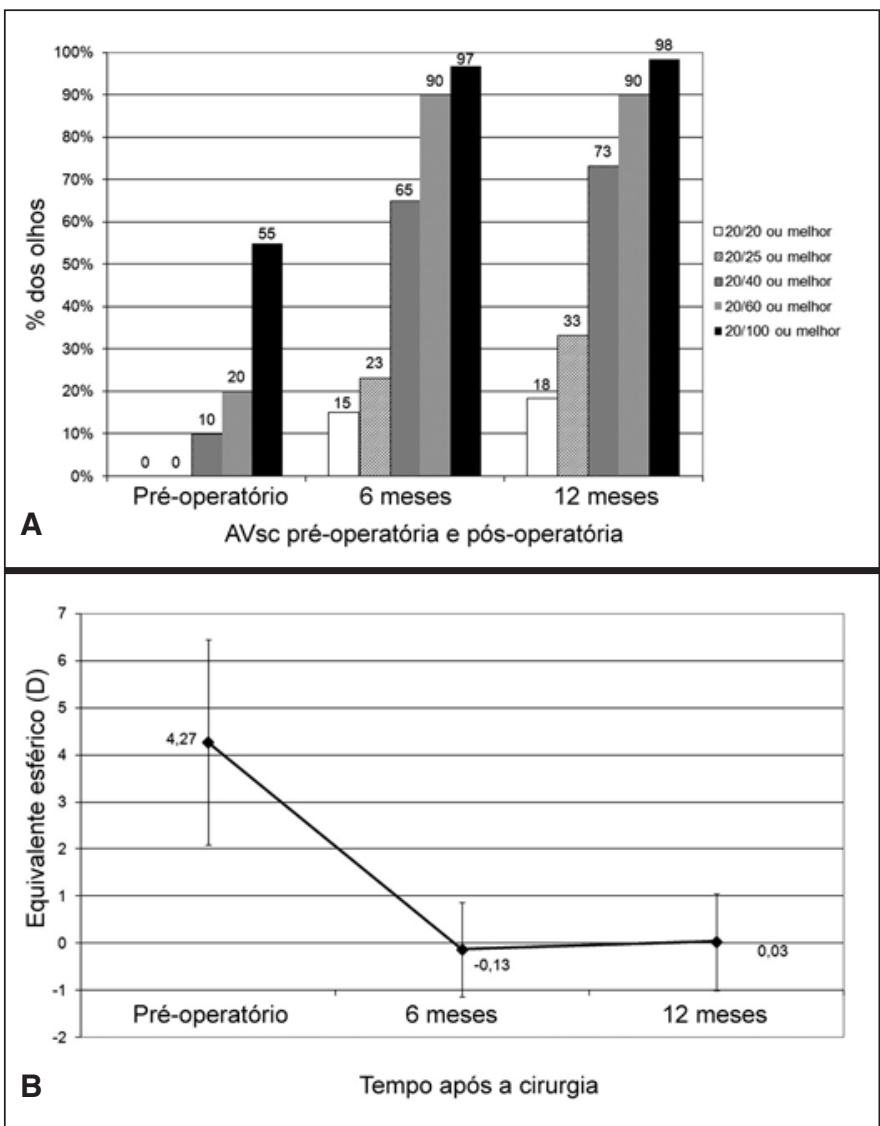

Figura 1 - A) Eficácia. Acuidade visual sem correção no pré-operatório e após seis e 12 meses do PRK guiado pela frente de onda corneana.

B) Estabilidade. Evolução do equivalente esférico ao longo do acompanhamento. Barras de erro representam \pm 1 desvio padrão.

Em relação a AVcc, após 1 ano, 35 olhos (58,3\%) haviam ganhado uma ou mais linhas de AVcc e 20 olhos $(33,3 \%)$, duas ou mais linhas. Somente um olho $(1,7 \%)$ perdeu duas linhas de AVcc (Figura 4).

Outras complicações incluíram três olhos que adquiriram ceratoconjuntivite adenoviral com formação de cicatrizes estromais e um olho que desenvolveu ectasia na média-periferia temporal inferior da córnea, causada por alargamento progressivo de uma incisão radial, após seis meses da fotoablação.

\section{DISCUSS ÃO}

A correção da hipermetropia após a CR é uma situação única e desafiadora por envolver córneas frequentemente irregulares e algumas vezes instáveis, com altas ametropias, o que pode levar a piores resultados visuais e refrativos do que em olhos sem cirurgias prévias. O uso do excimer laser para tratamento desses casos foi primeiramente descrito em 1997, em um estudo retrospectivo envolvendo 10 olhos, aonde se utilizou o PRK ${ }^{(19)}$.

A partir de 1999 predominou o uso do H-LASIK após a CR por proporcionar recuperação visual mais rápida, indolor e com 
74 Ceratectomia fotorrefrativa com mitomicina-C personalizada pela frente de onda corneana para o tratamento de hipermetropia após ceratotomia radial

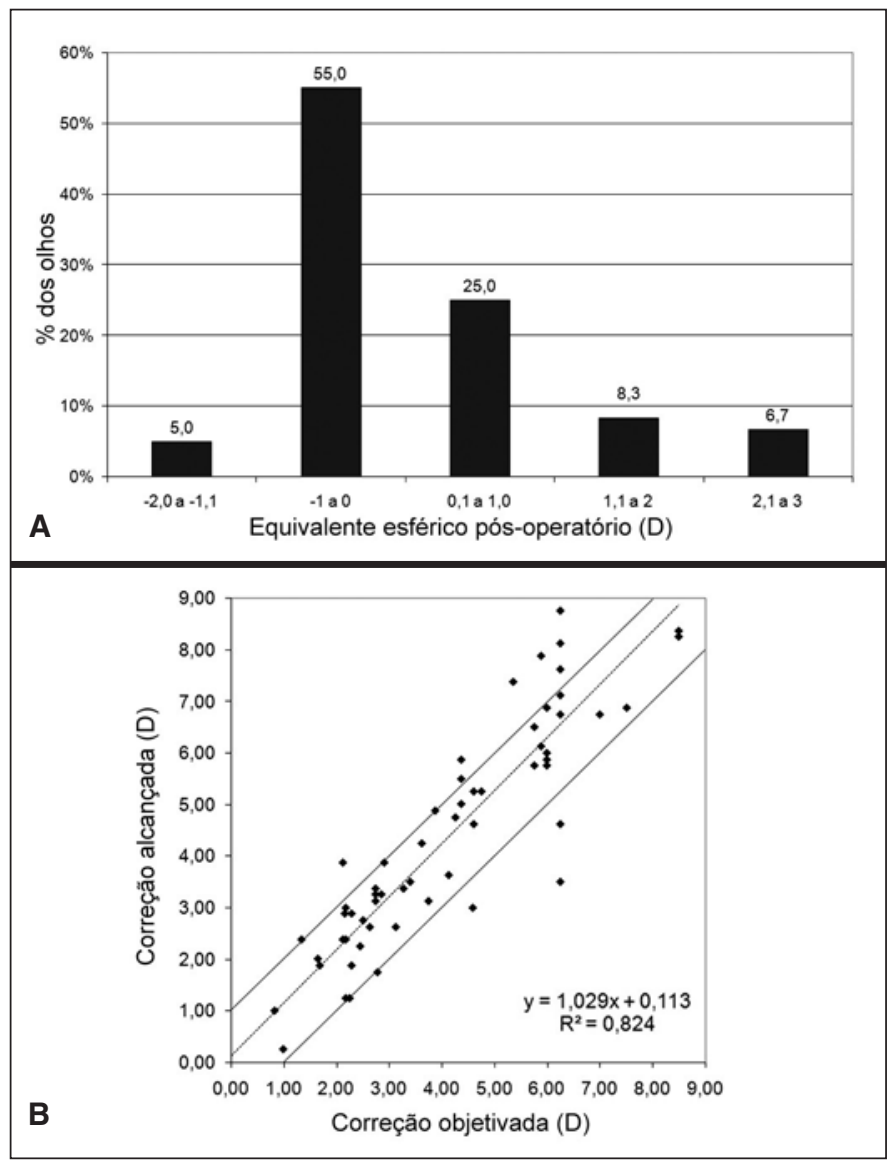

Figura 2 - A) Previsibilidade do equivalente esférico após um ano do PRK guiado pela frente de onda corneana; B) Gráfico de dispersão ilustrando a correção planejada vs. alcançada após um ano do tratamento

menor risco de "haze". Em 2002, entretanto, alguns autores publicaram os resultados de 69 olhos tratados com H-LASIK após $\mathrm{CR}^{(9)}$. Apesar de apresentar bons resultados refrativos, o número de complicações foi elevado, incluindo abertura das incisões radiais durante o levantamento do retalho, ceratite difusa lamelar e invasão epitelial da interface, que em um caso evoluiu para necrose do retalho, que foi removido. Outros estudos seguiram e atualmente, considera-se que o H-LASIK após CR apresenta um risco significativo de complicações ${ }^{(7-8,10-15)}$.

Nos primeiros estudos que utilizaram o PRK para corrigir miopia residual após CR observou-se maior incidência de "haze" central, regressão do resultado refrativo e perda de AVcc e sensibilidade de contraste ${ }^{(16,18)}$. Mas, para a correção de hipermetropia após CR, alguns autores, obtiveram bons resultados visuais e baixa incidência de "haze" com o PRK ${ }^{(17,19)}$.

No Brasil, alguns autores mostraram bons resultados com o PRK guiado pela topografia para a correção de hipermetropia e de astigmatismo após $\mathrm{CR}^{(3-4)}$. Em um estudo envolvendo 19 olhos foi usada MMC intraoperatória a 0,02\% para diminuir a incidência de "haze"(3). Porém os autores observaram, ainda assim, formação de "haze" periférico e central em alguns olhos com altas ametropias.
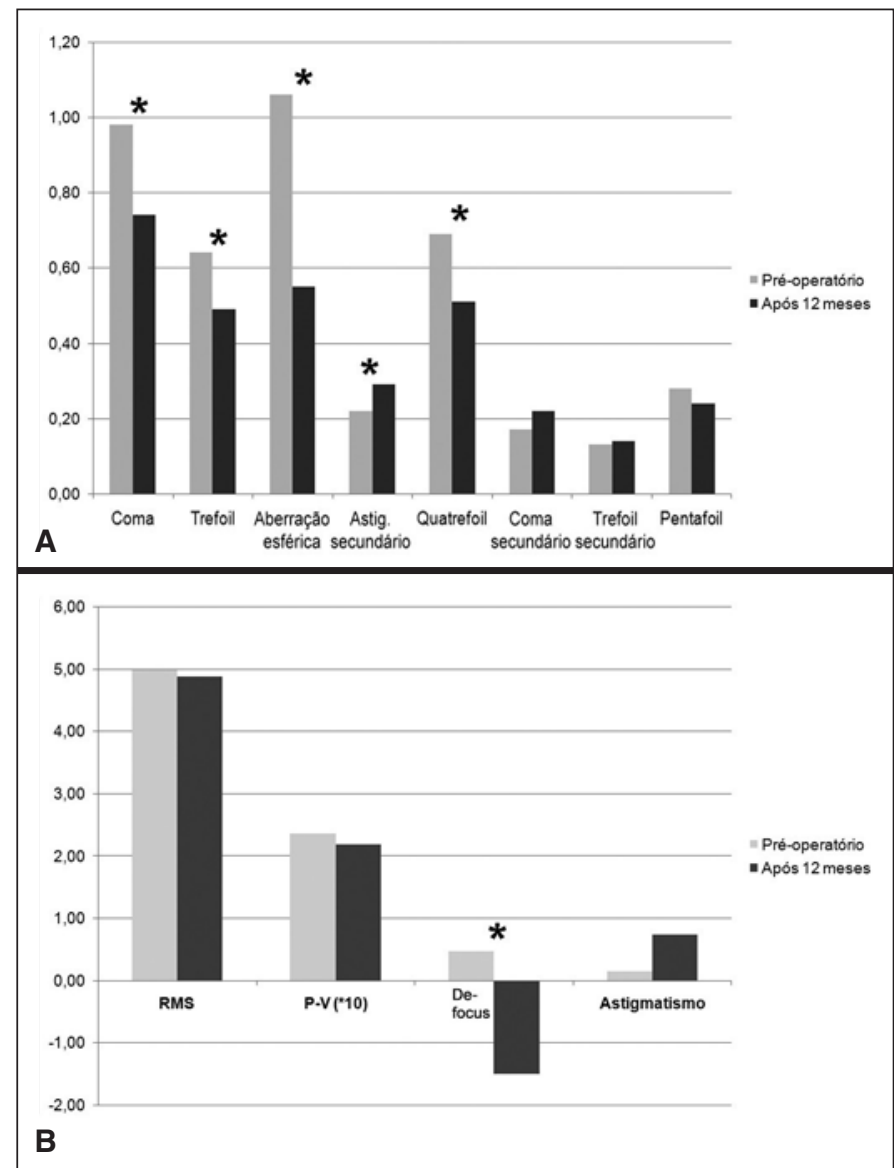

Figura 3 - A) Evolução do RMS, valor P-V e aberrações corneanas de baixa ordem. Asteriscos representam diferenças estatisticamente significantes; B) Evolução das aberrações corneanas de alta ordem. Asteriscos representam diferenças estatisticamente significantes.

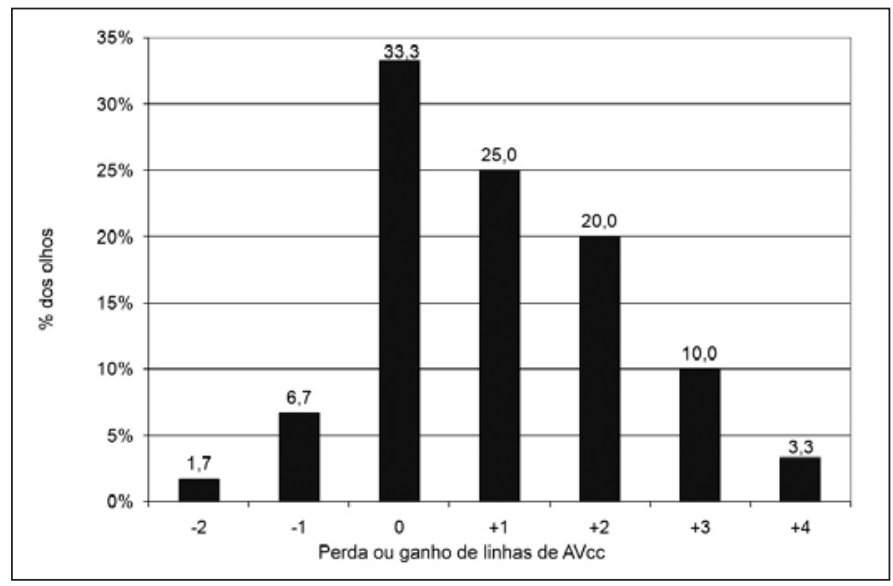

Figura 4 - Modificação da acuidade visual corrigida um ano após o tratamento

No presente estudo decidiu-se, também, usar MMC por se tratar de fotoablações personalizadas, mais profundas que as convencionais, e pela presença de cirurgia corneana prévia, fatores que aumentam o risco de "haze"(3,20-22). 


\section{Resultados visuais e refrativos}

Observou-se uma redução estatisticamente significativa da hipermetropia e do astigmatismo após 12 meses.

Os resultados refrativos comparam-se favoravelmente aos de outros estudos, com redução do EE médio de $+4,27$ para $+0,04,80 \%$ com EE entre $\pm 1,00 \mathrm{D}$ do planejado e $73 \%$ dos olhos alcançando AVsc de 20/40 ou melhor. Por exemplo, em um estudo com 69 olhos submetidos a LASIK para hipermetropia após CR, obteve-se redução do EE médio de $+3,40$ para -0,35 D, com 65\% dos olhos alcançando AVsc de 20/40 ou melhor e $80 \%$ com EE entre $\pm 1,00 \mathrm{D}^{(9)}$. Outros estudos utilizando LASIK para baixas e médias hipermetropias demonstraram melhores resultados: AVsc de 20/40 ou melhor em $86 \%$ dos olhos $^{(13)}$; em $90 \%(11)$; e em $83 \%{ }^{(10)}$. Estes estudos, contudo, apresentaram os resultados finais, incluindo pacientes submetidos a retratamentos.

\section{Aberrometria corneana}

Enquanto córneas normais costumam apresentar aberração esférica positiva de $0,28 \mu \mathrm{m}^{(25)}$, a média encontrada no préoperatório foi de $+1,06 \mu \mathrm{m}$, demonstrando o excessivo aplanamento central que ocorre nesses casos. Após o PRK a aberração esférica foi reduzida para $+0,55 \mu \mathrm{m}(P<0,001)$, principalmente pelo aumento da curvatura central corneana, alcançando um padrão mais próximo da normalidade, um fator importante na melhora da AVcc.

\section{CONCLUSÃO}

Nas condições deste estudo ficou demonstrado que o PRK personalizado pela frente de onda corneana com uso da MMC foi eficaz, previsível, seguro e estável pelo período de um ano para a redução da hipermetropia após CR. Observou-se melhora significativa da AVsc, AVcc e aberrações corneanas no pósoperatório.

\section{ABSTRACT}

Purpose: To assess the efficacy, predictability and stability of corneal wavefront-guided photorefractive keratectomy (PRK) for correcting hyperopia and astigmatism after radial keratotomy. Methods: In a prospective study, 60 eyes of 36 consecutive patients were treated with corneal wavefront-guided PRK with $0.02 \%$ mitomycin-C using an Esiris-Schwind excimer laser. Corneal epithelium was mechanically removed. All patients were followed-up for 12 months. Results: The mean time between radial keratotomy and PRK was 18.4 years \pm 3.8 (SD); mean spherical equivalent (SE) before radial keratotomy was -4.35 diopters $(\mathrm{D}) \pm 1.55$. Before PRK, the mean sphere was $+5.00 \mathrm{D} \pm 2.28$, mean astigmatism was $-1.47 \mathrm{D} \pm 1.06$, mean $\mathrm{SE}$ was $+4.27 \mathrm{D} \pm 2.18$, and the mean best-corrected visual acuity (BCVA) was $0.174 \pm 0.139$ (logMAR). The planned laser SE correction was $+4.74 \mathrm{D} \pm 2.11$. No intraoperative complica- tions were noted. At 12 months, mean SE was $+0.04 \mathrm{D} \pm 1.03$ $(P<0.001)$, mean astigmatism was $-1.03 \pm 0.75 \mathrm{D}(P=0.015)$, mean UCVA was $0.265 \pm 0.197$, and mean BCVA was $0.079 \pm$ $0.105(P<0.001)$. There was a mean gain of 1 line of BCVA and 20 eyes $(33.3 \%)$ gained 2 or more lines. Only one eye lost 2 lines. A significant decrease in coma $(P=0.002)$, trefoil $(P=0.004)$, spherical aberration $(P<0.001)$ and quatrefoil $(P=0.002)$ was observed. Forty eight eyes $(80 \%)$ were within $\pm 1.0 \mathrm{D}$ of intended SE. Mean regression from 6 to 12 months was $+0.17 \pm$ 0.67 D. Conclusion: Corneal wavefront-guided PRK was effective, predictable and stable after one year of follow-up for the treatment of hyperopia after radial keratotomy. A significant improvement in UCVA, BCVA and corneal aberrations was obtained.

\section{ClinicalTrials.gov Identifier: NCT00917657}

Keywords: Photorefractive keratectomy; Mitomycin; Radial keratotomy; Hyperopia; Astigmatism

\section{REFERÊNCIAS}

1. Waring GO, $3^{\text {rd }}$, Lynn MJ, McDonnell PJ. Results of the prospective evaluation of radial keratotomy (PERK) study 10 years after surgery. Arch Ophthalmol 1994;112(10):1298-308.

2. Hemenger RP, Tomlinson A, Caroline PJ. Role of spherical aberration in contrast sensitivity loss with radial keratotomy. Invest Ophthalmol Vis Sci 1989; 30(9):1997-2001

3. Ghanem RC, Ghanem VC, de Souza DC, Kara-José N, Ghanem EA. Customized topography-guided photorefractive keratectomy with the MEL-70 platform and mitomycin-C to correct hyperopia after radial keratotomy. J Refract Surg 2008;24(9):911-22.

4. Ghanem VC, Ghanem RC, Ghanem EA, Souza DC, Souza GC. [Topographically-guided photorefractive keratectomy for the management of secondary hyperopia following radial keratectomy]. Arq Bras Oftalmol 2007;70(5):803-8.

5. Coral-Ghanem C, Kara-José N, Ghanem VC. Lentes de Contato Pós-Cirurgia Ceratorrefrativa. In: Alves MR, Chamon W, Nosé W, eds. Cirurgia Refrativa. Rio de Janeiro: Cultura Médica, 2003.

6. Damiano RE, Forstot SL, Frank CJ, Kasen WB. Purse-string sutures for hyperopia following radial keratotomy. J Refract Surg 1998;14(4):408-13.

7. Afshari NA, Schirra F, Rapoza PA, Talamo JH, Ludwig K, Adelman RA, et al. Laser in situ keratomileusis outcomes following radial keratotomy, astigmatic keratotomy, photorefractive keratectomy, and penetrating keratoplasty. J Cataract Refract Surg 2005;31(11):2093-100.

8. Clausse MA, Boutros G, Khanjian G, Wagner C, Garabet AL. A retrospective study of laser in situ keratomileusis after radial keratotomy. J Refract Surg 2001;17(2 Suppl):S200-1.

9. Francesconi CM, Nose RA, Nosé W. Hyperopic laser-assisted in situ keratomileusis for radial keratotomy induced hyperopia. Ophthalmology 2002;109(3): $602-5$.

10. Linebarger EJ, Hardten DR, Lindstrom RL. Laser-assisted in situ keratomileusis for correction of secondary hyperopia after radial keratotomy. Int Ophthalmol Clin 2000;40(3):125-32.

11. Lyle WA, Jin GJ. Laser in situ keratomileusis for consecutive hyperopia after myopic LASIK and radial keratotomy. J Cataract Refract Surg 2003;29(5):879-88.

12. Munoz G, Albarran-Diego C, Sakla HF, Javaloy J. Femtosecond laser in situ keratomileusis for consecutive hyperopia after radial keratotomy. J Cataract Refract Surg 2007;33(7):1183-9.

13. Oral D, Awwad ST, Seward MS, Bowman RW, McCulley JP, Cavanagh HD. Hyperopic laser in situ keratomileusis in eyes with previous radial keratotomy. J Cataract Refract Surg 2005;31(8):1561-8.

14. Chan CC, Rootman DS. Localized midperipheral corneal steepening after hyperopic LASIK following radial keratotomy. Cornea 2003;22(7):679-83.

15. Munoz G, Montes-Mico R, Albarran-Diego C, Alio JL. Keratectasia after bilateral laser in situ keratomileusis in a patient with previous radial and astigmatic keratotomy. J Cataract Refract Surg 2005;31(2):441-5.

16. Azar DT, Tuli S, Benson RA, Hardten DR. Photorefractive keratectomy for 
76 Ceratectomia fotorrefrativa com mitomicina-C personalizada pela frente de onda corneana

para o tratamento de hipermetropia após ceratotomia radial

residual myopia after radial keratotomy. PRK After RK Study Group. J Cataract Refract Surg 1998;24(3):303-11.

17. Joyal H, Gregoire J, Faucher A. Photorefractive keratectomy to correct hyperopic shift after radial keratotomy. J Cataract Refract Surg 2003;29(8):1502-6.

18. Ribeiro JC, McDonald MB, Lemos MM, Salz JJ, Dello Russo JV, Aquavella $\mathrm{JV}$, et al. Excimer laser photorefractive keratectomy after radial keratotomy. J Refract Surg 1995;11(3):165-9.

19. Venter JA. Photorefractive keratectomy for hyperopia after radial keratotomy. J Refract Surg 1997;13(5 Suppl):S456.

20. Carones F, Vigo L, Scandola E, Vacchini L. Evaluation of the prophylactic use of mitomycin-C to inhibit haze formation after photorefractive keratectomy. J Cataract Refract Surg 2002;28(12):2088-95.
21. Lacayo GO, $3^{\text {rd }}$, Majmudar PA. How and when to use mitomycin-C in refractive surgery. Curr Opin Ophthalmol 2005;16(4):256-9.

22. Majmudar PA, Forstot SL, Dennis RF, Nirankari VS, Damiano RE, Brenart R, et al. Topical mitomycin- $\mathrm{C}$ for subepithelial fibrosis after refractive corneal surgery. Ophthalmology 2000;107(1):89-94.

23. Fantes FE, Hanna KD, Waring GO, $3^{\text {rd }}$, Pouliquen Y, Thompson KP, Savoldelli M. Wound healing after excimer laser keratomileusis (photorefractive keratectomy) in monkeys. Arch Ophthalmol 1990;108(5):665-75.

24. Holladay JT. Visual acuity measurements. J Cataract Refract Surg 2004; 30(2):287-90

25. Wang L, Dai E, Koch DD, Nathoo A. Optical aberrations of the human anterior cornea. J Cataract Refract Surg 2003;29(8):1514-21.

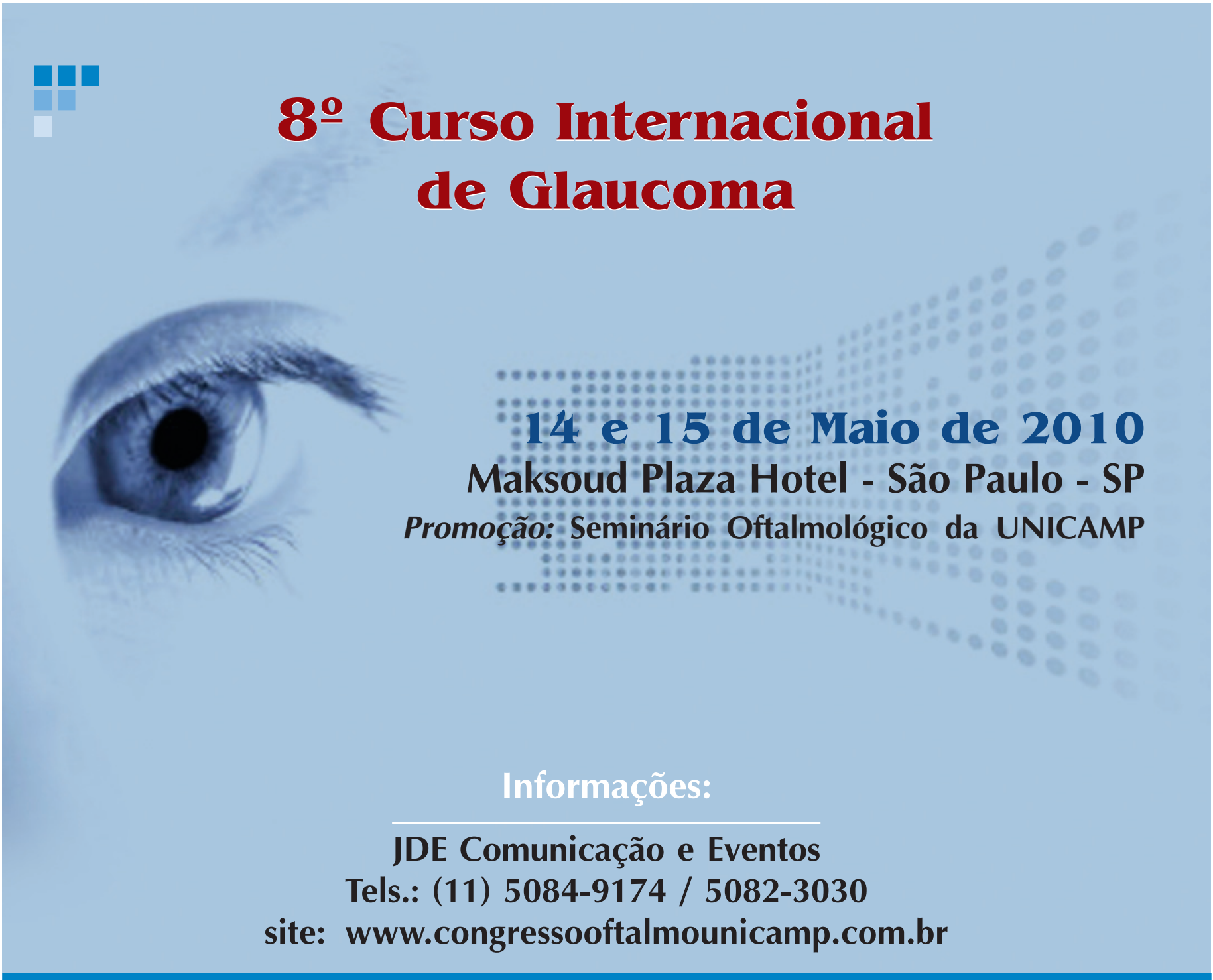

